\title{
COMPLEXITY CHANGES IN PRECLINICAL ALZHEIMER'S DISEASE: AN MEG STUDY OF SUBJECTIVE COGNITIVE DECLINE AND MILD COGNITIVE IMPAIRMENT
}

Authors: Elizabeth Shumbayawonda ${ }^{* 1}$, David López-Sanz ${ }^{* 2,3}$, Ricardo Bruña ${ }^{2,3,4}$, Noelia Serrano ${ }^{2,3}$, Alberto Fernández ${ }^{2,5}$, Fernando Maestú ${ }^{2,3,4}$ and Daniel Abasolo .

* These authors have contributed equally and thus share first authorship.

1 - Centre for Biomedical Engineering, Department of Mechanical Engineering Sciences, Faculty of Engineering and Physical Sciences, University of Surrey, Guildford, GU2 7XH, UK.

2 - Laboratory of Cognitive and Computational Neuroscience (UCM-UPM), Centre for Biomedical Technology (CTB), Technical University of Madrid (UPM), Madrid, Spain.

3 - Department of Experimental Psychology, Complutense University of Madrid, Madrid, Spain.

4 - Biomedical Research Networking Center in Bioengineering, Biomaterials and Nanomedicine, Zaragoza, Spain. 5 -Departamento de Medicina Legal, Psiquiatría y Patología, Complutense University of Madrid, Madrid, Spain. Corresponding author: David López Sanz.

Email: david.lopez@ctb.upm.es

\section{Abstract}

Objective: To analyse magnetoencephalogram (MEG) signals with Lempel-Ziv Complexity (LZC) to identify the regions of the brain showing changes related to cognitive decline and Alzheimer's Disease (AD).

Methods: LZC was used to study MEG signals in the source space from 99 participants (36 male, 63 female, average age: $71.82 \pm 4.06$ ) in three groups (33 subjects per group): healthy (control) older adults, older adults with subjective cognitive decline (SCD), and adults with mild cognitive impairment (MCI). Analyses were performed in broadband $(2-45 \mathrm{~Hz})$ and in classic narrow bands (theta $(4-8 \mathrm{~Hz})$, alpha (8$12 \mathrm{~Hz})$, low beta $(12-20 \mathrm{~Hz})$, high beta $(20-30 \mathrm{~Hz})$, and, gamma $(30-45 \mathrm{~Hz}))$

Results: LZC was significantly lower in subjects with MCI than in those with SCD. Moreover, subjects with MCI had significantly lower MEG complexity than controls and SCD subjects in the beta frequency band. Lower complexity was correlated with smaller hippocampal volumes.

Conclusions: Brain complexity - measured with LZC - decreases in MCI patients when compared to SCD and healthy controls. This decrease is associated with a decrease in hippocampal volume, a key feature in $\mathrm{AD}$ progression.

Significance: This is the first study to date characterising the changes of brain activity complexity showing the specific spatial pattern of the alterations as well as the morphological correlations throughout preclinical stages of AD.

Keywords: Lempel-Ziv Complexity, Magnetoencephalogram, Subjective Cognitive Decline, Mild Cognitive Impairment, Source Space, Alzheimer's disease

\section{Highlights}

- Brain complexity is decreased in the early stages of $\mathrm{AD}$, even before dementia onset.

- First source-level complexity analysis revealed impairment in key AD areas such as precuneus

- Complexity alterations were related to hippocampal atrophy, a hallmark of AD neurodegeneration 


\section{Funding}

This study was supported by a project from the Spanish Ministry of Economy and Competitiveness, PSI2009-14415-

C03-01 and a project by Comunidad de Madrid, B2017/BMD-3760 (NEUROCENTRO-CM). Elizabeth Shumbayawonda was supported by a Santander Universities Postgraduate Research Award.

Conflict of interest

The authors declare that the research was conducted in the absence of any commercial or financial relationships that could be construed as a potential conflict of interest. 


\section{Introduction}

Cognitive health, and its evolution with age, is an area of intense research, with one of the main drivers of this research being the increase in life expectancy in our modern day. Brain health is a difficult term to define as it covers a wide range of aspects, nevertheless, the Healthy Aging Research Network which is part of the Center for Disease Prevention and Control (CDC) in the United States has defined a healthy brain with preserved cognition as "one that can perform all the mental processes that are collectively known as cognition, including the ability to learn new things, intuition, judgement, language and remembering" (Centers for Disease Control and Prevention and Healthy Aging, 2007).

Ageing is typically associated with a decline in cognitive ability (Ardila et al., 2000). For some of the older adults this decline could be accompanied with a strong feeling of a more accentuated cognitive worsening compared to their age counterparts. This phenomenon is known as Subjective Cognitive Decline (SCD), and it could represent one of the earliest indicators of early neurodegeneration (Jessen et al., 2015). Recent studies into the development of dementia, particularly Alzheimer's Disease (AD), have suggested that there may be a link between SCD and AD (Rabin et al., 2017). This is partly due to the deviations from healthy ageing that have been observed in SCD patients as well as the similarities of the symptoms of SCD and AD. Nevertheless, although some studies such as those performed by Kwan (2017), López-Sanz et al. (2017b), and Rabin et al. (2017) have highlighted the subtle presence of $\mathrm{AD}$ biomarkers in SCD, at this stage $\mathrm{SCD}$ is currently largely undetectable by standard neuropsychological tests as its effects can be compensated (Erk et al., 2011).

The continued decline in cognitive ability can result in the development of MCI. MCI is a state characterised by a significant reduction in cognitive abilities affecting one or more domains. However, MCI patients cannot be classified as having dementia, as their independent functioning is still preserved (Petersen, 2004). As cognitive abilities continue to decline, studies have shown that MCI individuals are at a higher risk of suffering from AD than healthy individuals (Petersen and Negash, 2008; Landau et al., 2010; Petersen, 2011). Therefore, due to the numerous similarities between MCI and AD (Fischer et al., 2007; Vos et al., 2013), MCI and SCD (López-Sanz et al., 2017a), as well as SCD and $\mathrm{AD}$ (Rabin et al., 2017), it becomes important to investigate the progression and early onset of $\mathrm{AD}$ as it is the most prevalent form of dementia (accounting for $60-80 \%$ of reported cases (Barnes and Yaffe, 2011)). Additionally, there is a growing socioeconomic demand to provide care for fully demented patients. Thus, it is necessary to understand the development of cognitive decline in its early stages to develop a long-lasting solution to successfully combating dementia. Overtime these solutions can result in robust, efficient and successful early diagnosis and, therefore, will ease the burden on the socioeconomic, healthcare and scientific communities. Hence, it is with this in mind that this investigation was performed.

Magnetoencephalography is a non-invasive analysis technique used to record the magnetic fields generated by electrical activity in the human brain in a reference free manner (Gomez et al., 2008; Escudero et al., 2009; Jafarpour et al., 2013). The use of magnetoencephalograms (MEGs) to study the background activity of the brain has increased over the years due its advantages, including good temporal resolution, an adequate spatial resolution, particularly when combined with an individual MRI, good signal-to-noise ratio, and relatively robust against volume conduction when compared to other measures such as electroencephalography (Stam et al., 2007; 2016). Moreover, due to these advantages, the use of magnetoencephalography to investigate the early stages of AD has been 
recommended by the International Working Group (IWG) and the American Alzheimer's Association (Dubois et al., 2016). Therefore, in this study MEG recordings were used to investigate the changes in brain activity associated with cognitive decline.

Non-linear signal processing methods have successfully been used to investigate changes in the electrical and magnetic activity of the brain associated with various aspects such as age (Lutzenberger et al., 1995; Meyer-Lindenber, 1996; Shumbayawonda et al., 2017), sex (Li et al., 2014), and pathology (Li et al., 2014). One family of non-linear analysis methods focusses on the characterisation of complexity (Tononi et al., 1998). Complexity is a concept that can be used to assess changes in MEG brain activity to identify the direct/indirect effects of cortical functional organization of the brain (Li et al., 2014). Thus, complexity could be a robust indicator of the variances in cortical neuronal interaction as changes in complexity of the MEG signal may be coarsely related to the changes in the regularity of the interactions occurring within the neuronal network. It is also worth noting that, among the numerous complexity estimation techniques, those based on symbolic dynamic analysis are arguably the most computationally efficient. Lempel-Ziv Complexity (LZC) is a robust symbolic dynamic complexity technique that has been used to successfully investigate changes in electrophysiological activity associated with age (Li et al., 2014), AD (Gómez et al., 2006), schizophrenia (Fernández et al., 2011) or changes associated with cognitive decline (Fernández et al., 2012). LZC is a non-parametric method that can be used for the robust estimation of complexity of short time series and reflects the number of distinct substrings along a given sequence as well as their rate of recurrence (Fernandez et al., 2012). The LZC algorithm reflects the magnitude of the signal points and is frequency sensitive (Lempel and $\mathrm{Ziv}, 1976)$. Therefore, more complex or irregular time series would have greater LZC values than those featuring more regular patterns (Fernandez et al., 2012). In addition to this, when compared to other symbolic dynamic measures, LZC does not require the time series being analysed to be stationary. This is, therefore, a significant advantage when studying physiological signals (Shumbayawonda et al., 2018) and represents a major advantage of this metric. Nevertheless, previous MEG studies using LZC have analysed signals in the sensor space, but not in source space. The current study tries to address this limitation of previous work by extending the analysis of the complexity of MEG signals to the source space. It was hypothesised that LZC analysis in source space would reveal more specific information about the changes in MEG activity associated with cognitive decline in the preclinical stages of AD.

\section{Materials and methods}

\subsection{Subjects and diagnosis criteria}

Subjects were volunteers from three centres, namely the: Department of Neurology from Hospital Universitario San Carlos, Centre for Prevention of Cognitive Impairment, and Seniors Centre of Chamartin District in Madrid (Spain). A total of 99 subjects MEG recordings (36 males, 63 female) with an average age of $71.82 \pm 4.06$ made up the dataset used in this study as shown in Table 1. All subjects were age-matched, with no significant differences between the genders. All the subjects signed an informed consent and the procedures were approved by the local ethic committee of Hospital Universitario San Carlos.

Diagnosis of subjects was done in 3 steps. Firstly, a set of screening questionnaires including: the Mini Mental State Examination (MMSE; (Lobo et al., 1979), the 7 Minutes test, the Hachinski Ischemic Score (HIS; (Rosen et al., 1980), the Functional Assessment Questionnaire (Pfeffer et al., 1982), and the Geriatric Depression Scale-Short Form (Yesavage et al., 1982), were used to assess the general functional and 
cognitive status of each subject. After this, an exhaustive neuropsychological assessment ensued. This neuropsychological assessment included: Phonemic and Semantic Fluency (Controlled oral Word Association Test, COWAT), Direct and Inverse Digit Span Test (Wechsler Memory Scale, WMS-III), Immediate and Delayed Recall (WMS-III), Trail Making Test A and B (TMTA and TMTB; (Reitan, 1958)), Ideomotor Praxis of Barcelona Test, Boston Naming Test (BNT), and Rule Shift Cards (Behavioral Assessment of the Dysexecutive Syndrome, BADS).

Table 1: Demographic and neuropsychological data for each group. The analysis was done separately for the three diagnosis groups controls (CN), subjective cognitive decline (SCD), and Mild cognitive impairment (MCI). The left half of the table shows mean and standard deviation of demographic information, neuropsychological scores for each group. The right half of the table shows the p-values resulting of the ANOVA comparisons between groups. If the main factor was not significant (i.e. $p>0.05)$ hyphens are shown, otherwise the $p$-value for the pairwise comparisons are shown. Values shown in bold denote significant values ( $p$ <0.05). MMSE stands for Mini Mental State Examination. Trail Making Test B (TMTB).

\begin{tabular}{|c|c|c|c|c|c|c|}
\hline & \multicolumn{3}{|c|}{ Mean \pm SD } & \multicolumn{3}{|c|}{ p-values } \\
\hline & $\begin{array}{c}\mathrm{CN} \\
(\mathrm{N}=33)\end{array}$ & $\begin{array}{c}\text { SCD } \\
(\mathrm{N}=33)\end{array}$ & $\begin{array}{c}\text { MCI } \\
(\mathrm{N}=33)\end{array}$ & CN-SCD & CN-MCI & SCD-MCI \\
\hline Age & $71.85 \pm 3.96$ & $71.88 \pm 4.29$ & $71.72 \pm 3.91$ & - & - & - \\
\hline Gender (M-F) & $12-21$ & $12-21$ & $12-21$ & - & - & - \\
\hline MMSE & $28.90 \pm 1.25$ & $28.34 \pm 1.51$ & $26.18 \pm 2.84$ & 0.234 & $3.4 \times 10^{-7}$ & $2.3 \times 10^{-4}$ \\
\hline Direct digit & $8.37 \pm 1.89$ & $8.67 \pm 2.29$ & $7.68 \pm 1.83$ & 0.366 & 0.092 & 0.002 \\
\hline Inverse digit & $6.10 \pm 1.58$ & $5.70 \pm 1.83$ & $4.32 \pm 1.44$ & 0.915 & $7.3 \times 10^{-6}$ & $5 \times 10^{-5}$ \\
\hline TMTB & $\begin{array}{c}110.10 \pm \\
69.30\end{array}$ & $146.50 \pm 66.96$ & $218 \pm 103.32$ & 0.605 & $1.6 \times 10^{-7}$ & $6.7 \times 10^{-6}$ \\
\hline
\end{tabular}

The final group allocation was made after clinical evaluation with multidisciplinary consensus (by neuropsychologists, psychiatrists, and neurologists). For the SCD group, diagnosis was established after ensuring normal cognitive performance. Self-perceived cognitive decline was reported by the participants in a clinical interview. Possible confounders of SCD such as psycho-affective problems, psycho-active drugs consumption or other possible relevant conditions were excluded from the sample. Moreover, all participants were older than 60 at onset of SCD, and the onset of SCD had to have occurred within the last 5 years, similarly to previous studies (López-Sanz et al., 2017b).

Lastly, MCI was diagnosed according to the criteria put forward by Petersen (2004) and Grundman (2004). Besides meeting the core clinical criteria for $\mathrm{MCI}$, further exclusion criteria used in the compilation of the sample making up the database used in this study included:

1) History or presence of any neurological/psychiatric disorder or drug consumption that could interfere with MEG activity.

2) Presence of infarction, infection, or lesions in a T2 MRI within 2 months before MEG scan.

3) A modified Hachinski score and GDS-SF score equal or greater than 5;

4) Chronic use of anxiolytics, narcotics, anticonvulsants, hypnotics or history of alcoholism.

5) Presence of HIV, thyroid disorders, deficit of B12 vitamin, diabetes mellitus or syphilis.

\subsection{MEG recordings}


MEG signals during resting state were recorded at the Laboratory of Cognitive and Computational Neuroscience (Madrid, Spain). Data were acquired using a Vectorview MEG system (MEGIN) consisting of 306 channels (204 planar gradiometers and 102 magnetometers), while subjects sat comfortably with eyes closed inside a magnetically shielded room (VacuumSchmelze GmbH, Hanau, Germany). During signal acquisition, we placed two head position indication (HPI) on the mastoids bilaterally and two more on the forehead to enable continuous head position estimation, allowing tracking head movements. Moreover, a vertical electrooculogram of the left eye was used to capture blinks and eye movements. MEG data were acquired using a sampling rate of $1,000 \mathrm{~Hz}$ and an online anti-alias band-pass filter $(0.1$ and $330 \mathrm{~Hz})$.

\subsection{MEG signal pre-processing}

In the first step, MEG recordings were processed to remove magnetic noise originated outside the head with a spatiotemporal signal space separation algorithm (Taulu and Simola, 2006) using as parameters a correlation window of 0.9 and a time window of $10 \mathrm{~s}$. Signal space separation (SSS) filter decomposes MEG activity in different components originated inside and outside the head, and by removing the latter reduce the noise in the signals captured by MEG sensors. During pre-processing, an automatic algorithm from the Fieldtrip package (Oostenveld et al., 2011) was used to identify jump, blink and muscular artefacts. Afterwards automatic artefacts were visually inspected. The remaining data were segmented in 4 second epochs of artefact free activity. Subjects with at least 15 clean epochs were selected for further analysis, resulting in 47.6 (SD: 7.3) epochs in the healthy control (CN) group, 46.2 (SD: 9.4) epochs in the SCD group, and 42.2 (SD: 7.0) epochs in the MCI group. Furthermore, an ICAbased algorithm was employed to discard the electrocardiographic contribution. The application of tSSS filter leads to signal redundancy between the two types of MEG sensors, and thus only magnetometers data were used in the analysis (Garcés et al., 2017). 2 seconds of real signal on both sides were used as padding prior to the application of a $1800^{\text {th }}$ order finite impulse response band-pass filter between 2 and $45 \mathrm{~Hz}$ to prevent edge artefacts. A two-pass filtering procedure with a Hamming window was used in order to avoid phase distortion.

\subsection{MRI acquisition}

Each subject underwent a T1-weighted MRI. Participants were scanned using a General Electric 1.5 Tesla MR, with a high-resolution antenna and a homogenization PURE filter (Fast Spoiled Gradient Echo sequence, TR/TE/TI $=11.2 / 4.2 / 450 \mathrm{~ms}$; flip angle $12^{\circ} ; 1 \mathrm{~mm}$ slice thickness, $256 \times 256$ matrix and FOV $25 \mathrm{~cm}$ ). In order to acquire the volumes of several brain regions, the specialised automated tool for segmentation available in the FreeSurfer software (version 5.1.0) was used during pre-processing stages. Moreover, due to their association with $\mathrm{MCI}$ and AD progression, medial temporal structures' volumes were selected for use as anatomical evidence of brain atrophy and included in the correlation analyses carried out in the manuscript, to further elucidate the meaning of the electrophysiological findings ${ }^{2}$. All brain volumes were normalized dividing them by the total intracranial volume (ICV), so that total head volume did not influence the results.

\subsection{Source reconstruction}

To construct the source model, we used a modified version of the Harvard-Oxford atlas with 1485 cortical sources located in the vertices of a $1 \mathrm{~cm}$ homogeneous grid in MNI template, after which each source was linearly transformed into subject space. Following the linear transformation, each source belonging to one of the 64 areas of the reduced Harvard-Oxford atlas was labelled. The lead field was 
then calculated computed using a realistic single shell model (Nolte 2003). Lastly, the source time series were reconstructed by means of a Linearly Constrained Minimum Variance (LCMV) beamformer 4 using the aforementioned lead field and the epoch-averaged covariance matrix.

\subsection{Lempel-Ziv Complexity}

Complexity is a measure that is used to solve information theoretic problems in coding, and, data compression (Fernandez et al., 2012). LZC calculates the complexity of a signal in Kolmogorov's sense after it has been coarse-grained into a finite symbol sequence. For robustness to arbitrary outliers, most biological signals are transformed into a binary sequence using the median as the threshold $\left(T_{d}\right)$ in the coarse-graining process (Aboy et al., 2006). Therefore, in this study, the median was used as the threshold (Fernández et al., 2011; 2012; Abasolo et al., 2015). Furthermore, using the median as the threshold, ensures an equal number of zeroes and ones in the signal reducing the possible bias in LZC that could be introduced by using other thresholds. The LZC of a time series can be determined as follows: firstly, the signal $x(i)$ is transformed into a binary string $s(i)$ :

$$
s(i)=\left\{\begin{array}{rr}
0, & \text { if } x(i)<T_{d} \\
1, & \text { otherwise }
\end{array}\right.
$$

The binary string $s(i)$ is then scanned from left to right and a complexity counter $c(n)$ is increased by one unit every time a new subsequence of consecutive characters is encountered in the scanning process. The complexity counter $c(n)$ has an upper bound given by:

$$
c(n)<\frac{n}{\left(1-\epsilon_{n}\right) \log _{\alpha}(n)}
$$

where $\alpha$ is the base of the logarithm and $\epsilon_{n}$ is a small quantity such that $\epsilon_{n} \rightarrow 0$ when $n \rightarrow \infty$. Thus, the upper limit for $c(n)$ becomes $b(n)$ (Aboy et al., 2006; Fernandez et al., 2012):

$$
\lim _{n \rightarrow \infty} c(n)=b(n)=\frac{n}{\log _{\alpha}(n)}
$$

and in the binary case considered herein with two symbols used in the coarse-graining process of the original signal:

$$
b(n)=\frac{n}{\log _{2}(n)}
$$

The complexity counter $c(n)$ can be normalised using $b(n)$ to give $C(n)$ which reflects the rising number of new patterns in the sequence:

$$
C(n)=L Z C=\frac{c(n)}{b(n)}
$$

LZC is a univariate method and the normalised values are in the range $0 \leq L Z C \leq 1$ where the lower limit shows a stationary signal with no varying dynamics present, while the upper limit shows a very complex signal with multiple complex dynamics present (Aboy et al., 2006).

LZC has been used successfully to extract information from both MEG (Fernández et al., 2011; 2012) and EEG signals (Abasolo et al., 2015; Husseen Al-Nuaimi et al., 2018) over the years. In this study, LZC 
was estimated in both broadband $(2-45 \mathrm{~Hz})$ and narrow bands (like work done by Liu et al. (2016)), i.e. theta band $(4-8 \mathrm{~Hz})$, alpha $(8-12 \mathrm{~Hz})$, beta band (low beta $(12-20 \mathrm{~Hz})$, high beta $(20-30 \mathrm{~Hz}))$, and, gamma band $(30-45 \mathrm{~Hz})$. Furthermore, similarly to work done by López-Sanz et al. (2017a) and Cheng et al. (2017), the effect of the three diagnostic groups; controls (CN), subjective cognitive decline (SCD), and mild cognitive impairment (MCI) on brain activity complexity was evaluated.

\subsection{Statistics}

A first exploratory analysis was performed to find group differences between $\mathrm{CN}, \mathrm{SCD}$ and $\mathrm{MCI}$ average whole-head broadband complexity values using one-way ANOVA with diagnosis as between group factor, using IBM Statistical Package for the Social Sciences (SPSS) Statistical Data Editor version 24. Further statistical analyses to identify the cortical sources of the significant differences between groups were performed using independent $\mathrm{t}$-tests and the Monte-Carlo method, with corrections for multiple comparisons done using cluster-based permutation tests (CBPT) with 10,000 random data partitions (Maris and Ostenveld, 2007). Pearson's correlation coefficients (used because results followed a normal distribution) between the source complexity values and biological data (such as bilateral hippocampus, parahippocampus and entorhinal grey matter volumes) were also calculated for each source separately to identify associations between the LZC values and grey matter atrophy. By doing so, we calculated 1485 correlations (i.e. the number of cortical sources) between the corresponding complexity value and grey matter volume of each medial temporal structure included. Significant correlations were afterwards grouped in significant spatially contiguous clusters and corrected for multiple comparisons using CBPT. Additionally, to understand the relationship between LZC changes and cognition in the early stages of dementia in a better way, a correlation analyses using Pearson's coefficient between mean global complexity (i.e. LZC values obtained in the broadband analysis averaged across all sources) and neuropsychological tests were performed (results followed a normal distribution). This analysis was conceived as a merely exploratory analysis, therefore all the tests mentioned in section 2.1 employed for the diagnosis (excluding FAQ and Hachinski) were included to this purpose. Functional changes within the AD continuum do not always follow a linear trajectory in the different stages of the disease (Jones et al. 2015). Consequently, we decided to calculate correlations using our whole sample, but also in each group independently, so that we could detect specific relationships at each different phase of the disease.

\section{Results}

Statistical analysis using whole-brain broadband mean group LZC values for all the sources showed that only the differences between $\mathrm{SCD}$ and $\mathrm{MCI}$, (SCD-MCI) were statistically significant $(p=0.02)$, while the differences between controls and MCI groups (CN-MCI) and controls and SCD groups (CN-SD) were not ( $p=0.109$ and $p=0.468$, respectively),. When the average complexity values for each group were plotted on the same graph (see Fig 1), it was found that there was an inverted U relationship between complexity values and diagnosis group. Evidently, mean global LZC values for MCI subjects were lower compared to both healthy controls and SCD subjects, although only significantly with respect to the latter.

\section{\#\# Insert figure 1 about here \#\#}

Further statistical analyses using non-parametric CBPT between each of the individual cortical sources were then performed to identify the specific source localisation of the observed differences (in Fig 1). 
As shown in Fig 2, for MCI subjects the cortical sources in the posterior regions of the brain had lower LZC values when compared to the SCD group. Thus, to enable further understanding of the meaning of the results and their relation to changes in grey matter volume, correlations between complexity values and brain structure volume scores were evaluated. Results from these analyses showed that SCD values in the significant cluster were significantly correlated with the scores of the left parahippocampus $(r h o=0.397, p=0.024)$.

\section{\#\# Insert figure 2 about here \#\#}

Once the broadband analysis was complete, narrow band analyses ensued. By limiting the frequency band in which LZC is evaluated, subtle differences between the time series being analysed (which could have otherwise been hidden in broadband analyses) can be identified. Nevertheless, results from these narrowband analyses showed that only LZC values in the low beta band were significantly different, as shown in Fig 3. Moreover, a significant decrease in the MCI group with respect to both healthy controls $(p=0.0362)$ and SCD subjects $(p=0.0398)$ was found.

\section{\#\# Insert figure 3 about here \#\#}

Narrow band complexity analyses highlighted differences between the CN-MCI and the SCD-MCI groups unlike the broadband analyses, where significant differences between $\mathrm{CN}$ and $\mathrm{MCI}$ groups were not detected. In the comparison between $\mathrm{CN}$ and MCI, statistical differences ( $p=0.0362)$ were identified (in the low beta band) in the central regions of the brain, including relevant regions such as the anterior and posterior cingulate, precuneus, and, superior parietal lobe. The decrease in complexity was fairly symmetric between the two cerebral hemispheres of the brain. MCI subjects showed a decrease in complexity when compared with the SCD group in highly similar regions to those observed in the $\mathrm{CN}$ vs MCI comparison, although the differences between the SCD-MCI were slightly less symmetrically distributed. Nevertheless, like the results from the CN-MCI comparison, the statistically significant differences $(p=0.0398)$ between the complexity values for SCD and MCI were found in the low beta frequency band (12-20Hz) (Fig 3) and in regions including the precuneus and posterior cingulate.

Similarly, correlations between complexity values, and brain structure volume scores were also evaluated. Results showed that $\mathrm{CN}$ values in the significant cluster (in the low beta band) were significantly correlated with the volume of the right entorhinal cortex ( rho $=0.477, p=0.008$ ). Interestingly, as hypothesised, both correlations (for broad- and narrow- bands) highlight that lower values of complexity are related to lower medial temporal structure volumes.

Statistical analyses were then performed to identify the relationship between the changes in low beta LZC values and grey matter integrity. These analyses were performed using all the subjects $(C N, S C D$, and MCI) LZC values and hippocampal volumes. Results (Fig 4) highlighted a posterior cluster which was positively correlated $(p=0.0356)$ to the right-hippocampal volumetric scores, thus highlighting the association between higher low beta complexity values and brain structure volume scores.

\section{\#\# Insert figure 4 about here \#}

Regarding the correlation analysis between complexity and cognition, we observed that for the whole sample the average mean global LZC values (displayed in figure 1) did not show any significant association with the cognitive measurements included in the analysis. In contrast, group by group correlations highlighted significant correlations in some groups. For the healthy controls the time employed in solving both TMTa and TMTb were significantly and negatively associated to mean global 
complexity (TMTa_t, rho $=-0.35, p=0.048$; TMTb_t $r h o=-0.44, p=0.012$ ). Interestingly, we also observed significant trends of associations with MMSE ( $r h o=0.35, p=0.079)$, direct digits ( $r h o=0.32, p=0.073$ ) and 7-minute orientation subtest ( $r h o=0.29, p=0.09$ ). Among the SCD group total scores in the sevenminute test were negatively associated to mean global complexity ( $r h o=-0.37, p=0.033$ ). Furthermore, performance on the inverse digit test showed a strong tendency towards a negative correlation ( $r h o=-$ $0.33 ; p=0.06$ ) and TMTa completion time was marginally associated to mean global complexity ( $r h o=$ $0.3, p=0.089$ ). Remarkably, these results showed that while higher complexity values were associated to better cognitive performance in the healthy control group, the opposite was true for the SCD group. However, it is important to bear in mind the exploratory nature of this analysis. No significant correlations were found in the $\mathrm{MCI}$ group.

\section{Discussion}

The aim of this study was to evaluate changes between the brain activity from healthy controls, SCD and MCI recorded in MEG signals with LZC, a non-linear algorithm that quantifies complexity in time series. Our study found, for the first time in source space, significant alterations in non-linear brain dynamics in MCI patients. Particularly, MCI patients exhibited decreased broadband LZC with respect to subjects with SCD, who interestingly did not show significant alterations in these metrics. Narrow band analyses further confirmed MCI alterations, showing a specific decrease in low beta with respect to both healthy older adults and SCD in brain regions typically related to AD. Lastly, the lower complexity over posterior brain regions was associated to grey matter atrophy over medial temporal structures, which seems to support the pathological nature of our findings.

Broadband analyses showed that there was a significant decrease in complexity in MCI with respect to SCD participants. Moreover, it was also observed that although the SCD group had higher mean global LZC than the $\mathrm{CN}$ group, this difference was not statistically significant. Nevertheless, despite this, the finding relating decreased complexity within the $\mathrm{MCI}$ group is consistent with findings by Dauwels et al. (2010) in their evaluation of the effects of $\mathrm{AD}$, where observed decreases in complexity were associated with decreases in cognitive decline. Sporns et al. $(2004 ; 2005)$ noted that complexity is a measure that is strongly dependent on the underlying structure of the brain (i.e. neuroanatomy), while Fernandez et al. (2011) found that there were some correlations between LZC and fractal anisotropy (FA). These findings show that one of the drivers behind these complexity changes could be the modifications in the neuroanatomy making up the white matter of the brain. Considering the aforementioned evidence, it could be hypothesised that these complexity alterations are not yet detectable at the SCD stage given that complexity changes, apparently, rely heavily on, and are tightly related to, structural alterations, which are supposed to appear at a later stage than functional and synaptic disruption in the AD continuum (Jack Jr and Holtzman, 2013). In fact, although some studies have confirmed structural alterations in SCD (Peter et al., 2014) it is not absent from controversy, and some other studies (Tepest et al., 2008), including previous results on the sample from this study (LópezSanz et al., 2016) have not found evidence of any structural damage at this stage yet. Interestingly, our results also show that there is a relationship between complexity alterations and grey matter atrophy since complexity values were positively associated with the integrity of key structures in the development of $\mathrm{AD}$ such as medial temporal lobe regions.

The results from the analyses on narrow frequency bands in this study have also shown that, when compared to the $\mathrm{CN}$ and SCD groups, MCI subjects exhibit a marked decrease in complexity in the low 
beta band in sources located in the central and posterior regions of the brain. A previous study by López-Sanz et al (2016) also showed alterations in the MCI group in their spectral profile, characterised in that case by a significant reduction in the relative power in the alpha band. This correlates with suggestions put forward by studies done by Mormino et al. (2011), Buckner et al. (2009), and Johns et al. (2015), where it was found that AD-related alterations could be interpreted as being caused by either inefficient noisy synaptic transmission or by a compensation mechanism. The brain undergoes some kind of rewiring due to cognitive decline, as evidenced by the hyper- and hypo- connectivity patterns present in pre-dementia stages (Johns et al 2015, López et al 2017). Therefore, it is possible that only those individuals whose compensation stage is not enough to contain the initial effects of cognitive decline go on to develop MCI (Cheng et al., 2017). Moreover, although not significant, a slight increase in mean global complexity in the SCD group with respect to healthy controls is yet observable. Previous studies have linked increases in functional connectivity (FC), or in activation as a sign of compensation in early stages of dementia (Liang et al. 2011), and this could apparently be supported by correlations in our whole sample linking more preserved parahippocampal structures to higher complexity values. However, an interesting effect appeared when taking a closer look at the data. While healthy controls seemed to benefit from higher complexity values, i.e. those with more complex brain activity showed better cognitive performance, the opposite was true in the SCD group, whose participants showed negative associations between mean global complexity and cognitive scores. Similar results have been also commonly reported, where an increase in a certain metric, for instance FC (López-Sanz et al., 2017a) or alpha power (Nakamura et al., 2018), only reflects a pathological consequence of the disease and not a compensatory sign. In fact, although the cross-sectional nature of our study does not allow for chronological interpretations, our results could suggest a non-linear trajectory of the alterations in SCD. Findings from previous studies have shown that increases and decreases of metric values (such as FC (Jones et al., 2016; López-Sanz et al., 2017a)) are a common feature of the network failure which begins in the pre-dementia stages (SCD and MCI) and progresses along the AD continuum. Thus, in line with this, the results from this study showing a minor, not significant, increase in complexity followed by a significant decrease in complexity due to $\mathrm{MCI}$ add value to these findings as they show that brain activity complexity could follow a similar trajectory. However, it is important to bear in mind that due to the absence of statistical significance of this increase, no strong statements could be made in this regard based on the present results.

Regions showing lower complexity in the MCI group were of particular interest. A significant decrease in brain activity complexity in parietal and postero-medial regions, including the precuneus and posterior cingulate, was found. The precuneus and posterior cingulate are critically involved in the pathogenesis of $\mathrm{AD}$ and are key structures in the development of the disease (Ryu et al., 2010; Berti et al., 2010; Karas et al., 2007). Interestingly, these regions are one of the main hubs of the brain network, and a critical hotspot of the default mode network (Utevsky et al., 2014) These regions are known to be heavily affected in the early stages of the disease by hypometabolism, disconnection from other regions of the brain (Gili et al., 2011), and high A $\beta$ accumulation (Wong et al., 2010) among other alterations. Of note, the topographic distribution of this complexity alterations in the MCI patients (mainly affecting parietal regions), and the specific frequency range affected (i.e. low beta band), is in clear agreement with previous work revealing eigenfrequencies (or natural frequencies) of different brain regions (Rosanova et al., 2009). According to their results, TMS perturbation consistently produced low beta band oscillations over the above-mentioned brain regions, showing a natural tuning of these cortical circuits to oscillate in that frequency range. A posterior study proved that the enhancement of these 
natural frequencies using TMS may be a relevant tool able to modify cognitive performance (Romei $e t$ al., 2012). Our study showed for the first time that these regions present significant alterations in the complexity of their electrophysiological patterns even in the prodromal stages of $\mathrm{AD}$, the MCI stage, which could suggest an early alteration in the natural tuning of the oscillatory mechanisms in these cortical regions.

After exploring the between groups differences in complexity, an investigation to evaluate the possible correlation of these changes with differences in the volumes of some brain structures was performed. Positive correlations between complexity values and grey matter volume areas associated with memory and cognition were obtained in both broadband and narrow band analyses. Interestingly, grey matter atrophy over medial temporal structures is one of the key features of the AD pathological cascade (Cantero et al., 2016; Fox et al., 1996), and is highly associated to memory loss and progression throughout the continuum of the disease (Apostolova et al., 2006). In this study, results showed a significant association between lower complexity values over a posterior portion of the brain and smaller brain volume in the right parahippocampal regions. Previous studies have also shown a relationship between electrophysiological cortical activity and grey matter atrophy with respect to alpha power (Babiloni et al., 2015). Therefore, the results from this study can be interpreted as supporting our hypothesis that decreased complexity values in MCI are reflective of a pathological process related to $\mathrm{AD}$.

One of the main limitations of this study is the absence of AD biomarkers in our sample. Even though widely established clinical criteria were followed for the diagnosis of SCD and $\mathrm{MCI}$, we cannot be sure about the specific subgroups of subjects that will progress to develop dementia due to AD. Furthermore, the cross-sectional nature of our data limits our ability to properly interpret the evolution across the different preclinical stages, and thus longitudinal studies are needed in this regard. Lastly, even though 99 subjects were included in this study, which represents a relevant sample size considering current MEG literature in the field, future studies are required to replicate and validate these findings in independent and larger samples to increase the validity and generalisability of the results. Furthermore, results regarding the relationship between complexity changes and cognition are purely exploratory and should be validated in future studies. Moreover, future work investigating the use of other complexity and entropy measures in source space such as permutation LZC (PLZC) (Bai et al., 2015) and complexity index (CI) (Dimitriadis, 2018) is needed to increase our knowledge about the pathological nature associated with cognitive decline and to replicate current findings in the source space, where no other works other than this are available to date.

\section{Conclusions}

In conclusion, results showed that $\mathrm{MCI}$ was associated with a significant decrease in complexity (measured by means of LZC) when compared to controls and subjects with SCD. Moreover, these changes in LZC values were significantly correlated to decreases in right hippocampal volumes. Therefore, the outcomes from this study show, for the first time, the relationship between source space complexity and changes in prodromal stages of Alzheimer's disease.

\section{References}

Abasolo, D., Simons, S., Morgado da Silva, R., Tononi, G., and Vyazovskiy, V. V., 2015. Lempel-Ziv complexity of cortical activity during sleep and waking in rats. J Neurophysiol, Volume 113, p. 2742-2752. 
Aboy, M., Hornero, R., Abasolo, D. and Alvarez, D., 2006. Interpretation of Lempel ziv complexity measure in the context of biomedical signal analysis. IEEE Trans. Bio-Med. Eng, 53(11), pp. 2282-2288.

Apostolova, L. G., Dutton, R. A., Dinov, I. D., Hayashi, K. M., Toga, A. W., Cummings, J. L., and Thompson, P. M., 2006. Conversion of mild cognitive impairment to Alzheimer disease predicted by hippocampal atrophy maps. Arch Neurol, 63(5), pp. 693-9.

Ardila, A., Ostrosky-Solis, F. R. M. and Gómez, C., 2000. Age-Related Cognitive Decline During Normal Aging: The Complex Effect of Education. Arch. Clin. Neuropsych, 15(6), pp. 495-513.

Babiloni, C., Del Percio, C., Boccardi, M., Lizio, R., López , S., Carducci, F., et al., 2015. Occipital sources of restingstate alpha rhythms are related to local gray matter density in subjects with amnesic mild cognitive impairment and Alzheimer's disease. Neurobiol. Aging, 36(2), pp. 556-70.

Bai Y, Liang Z and Li X., 2015. A permutation Lempel-Ziv complexity measure for EEG analysis. Biomed Signal Process Control, 19:102-14.

Barnes, D. E. and Yaffe, K., 2011. The projected effect of risk factor reduction on Alzheimer's disease prevalence. Lancet Neurol, 10(9), pp. 819-28.

Berti, V., Osorio, R. S., Mosconi, L., Li, Y., De Santi, S., and de Leon, M. J., 2010. Early detection of Alzheimer's disease with PET imaging. Neurodegener. Dis, 7(1-3), pp. 131-5.

Cantero, J. L., Iglesias, J. E., Van Leemput, K. and Atienza, M., 2016. Regional Hippocampal Atrophy and Higher Levels of Plasma Amyloid-Beta Are Associated With Subjective Memory Complaints in Nondemented Elderly Subjects. J Gerontol A Biol Sci Med Sci 71(9), pp. 1210-5.

Centers for Disease Control and Prevention and Healthy Aging, 2007. What is a Healthy Brain? New Research Explores Perceptions of Cognitive Health Among Diverse Older Adults. [Online] Available at: https://www.cdc.gov/aging/pdf/perceptions of cog hlth factsheet.pdf [Accessed 31 May 2018].

Cheng, Y., Chen, T. and Chiu, M., 2017. From mild cognitive impairment to subjective cognitive decline: conceptual and methodological evolution. Neuropsychiatr Dis Treat, Volume 13, pp. 491-498.

Dauwels, J., Vialatte, F. and Cichocki, A., 2010. Diagnosis of Alzheimer's disease from EEG signals: where are we standing? Curr Alzheimer Res, 7(6), pp. 487-505.

Dimitriadis, S.I., 2018. Complexity of brain activity and connectivity in functional neuroimaging. J Neurosci Research, 96(11), pp. 1741-1757.

Dubois, B., Hampel, H., Feldman, H. H., Scheltens, P., Aisen, P., Andrieu, S., et al., 2016. Preclinical Alzheimer's disease: Definition, natural history, and diagnostic criteria.. Alzheimers Dement, 12(3), p. 292-323.

Erk, S., Spottke, A., Meisen, A., Wagner, M., Walter, H., and Jessen, F., 2011. Evidence of neuronal compensation during episodic memory in subjective memory impairment. Arch Gen Psychiatry, 68(8), pp. 845-52.

Escudero, J., Hornero, R., Abasolo, D. and Fernandez, A., 2009. Blind source separation to enhance spectral and non-linear features of magnetoencephalogram recordings. Application to Alzheimer's disease. Med Eng Phys, Volume 31, pp. 872-879.

Fernández, A., Gomez, C., Hornero, R. and López-Ibor, J., 2012. Complexity and schizophrenia. Prog. NeuroPsychopharmacol. Biol. Psychiatry, Volume 45, pp. 267-76.

Fernández, A., López-Ibor, M., Turrero, A., Santos, J., Morón, M., Hornero, R., et al., 2011. Lempel-Ziv complexity in schizophrenia: a MEG study. Clin Neurophysiol , 122(11), pp. 2227-35.

Fernandez, A., Rios-Lago, M., Abasolo, D., Hornero, R. A.-L., Paul, N., Maestu, F., and Ortiz, T., 2011. The correlation between white-matter microstructure and the complexity of spontaneous brain activity: A difussion tensor imaging-MEG study. NeuroImage, 57(4), pp. 1300-1307. 
Fernandez, A., Zuluaga, P., Abasolo, D., Gomez, C., Serra, A., Mendez, M., and Hornero, R., 2012. Brain oscillatory complexity across the life span.. Clin Neurophysiol, 123(11), pp. 2154-2162.

Fischer, P., Jungwirth, S., Zehetmayer, S., Weissgram, S., Hoenigschnabl, S., Gelpi, E., et al., 2007. Conversion from subtypes of mild cognitive impairment to Alzheimer dementia. Neurology, 68(4), p. 288-91.

Fox, N. C., Warrington, E. K., Freeborough, P. A., Hartikainen, P., Kennedy, A. M., Stevens, J. M., and Rossor, M. N., 1996. Presymptomatic hippocampal atrophy in Alzheimer's disease. A longitudinal MRI study.. Brain, 119(Pt 6), pp. 2001-7.

Garcés, P., López-Sanz, D., Maestú, F. and Pereda, E., 2017. Choice of Magnetometers and Gradiometers after Signal Space Separation. Sensors , 17(12), pp. 1-13.

Gili, T., Cercignani, M., Serra, L., Perri, R., Giove, F., Maraviglia, B., et al., 2011. Regional brain atrophy and functional disconnection across Alzheimer's disease evolution.. J. Neurol. Neurosurg. Psychiatry 82(1), pp. 58-66.

Gómez, C., Hornero, R., Abásolo, D., Fernández, A., and López, M., 2006. Complexity analysis of the magnetoencephalogram background activity in Alzheimer's disease patients. Med Eng Phys, 28(9), pp. 851-9.

Gomez, C., Hornero, R., Mediavilla, A., Fernandez, A., and Abasolo, D., 2008. Nonlinear forecasting measurement of magnetoencephalogram recordings from alzheimers disease patients. British Columbia, IEEE EMBS, pp. 2153-2156.

Grundman, M., Petersen, R. and Ferris, S. H., 2004. Mild Cognitive Impairment Can Be Distinguished From Alzheimer Disease and Normal Aging for Clinical Trials. Arch Neurology , 61(1), pp. 59-66.

Husseen Al-Nuaimi, A., Jammeh, E., Sun, L. and Ifeachor, E., 2018. Complexity Measures for Quantifying Changes in Electroencephalogram in Alzheimer's Disease. Complexity, 2012(8915079), pp. 1-12.

Jack Jr, C. R. and Holtzman, D. M., 2013. Biomarker modeling of Alzheimer's disease. Neuron, 80(6), pp. 1347-1358.

Jafarpour, A., Barnes, G., Fuentemilla, L., Duzel, E., and Penny, W. D., 2013. Population Level Inference for Multivariate MEG Analysis. PLoS, 8(8), pp. 1-8.

Jessen, F., Amariglio, R. E., van Boxtel, M., Breteler, M., Ceccaldi, M., Chételat, G., et al., 2015. A conceptual framework for research on subjective cognitive decline in preclinical Alzheimer's disease. Alzheimers Dement, 10(6), pp. 844-52.

Jones, D. T., Knopman, D. S., Gunter, J. L., Graff-Radford, J., Vemuri, P., Boeve, B. F., et al., 2016. Cascading network failure across the Alzheimer's disease spectrum. Brain, 139(Pt 2), pp. 547-62.

Karas, G., Scheltens, P., Rombouts, S., van Schijndel, R., Klein, M., Jones, B., et al., 2007. Precuneus atrophy in earlyonset Alzheimer's disease: a morphometric structural MRI study. Neuroradiology, 49(12), pp. 967-76.

Landau, S. M., Harvey, D., Madison, C. M., Reiman, E. M., Foster, N. L., Aisen, P. S., et al., 2010. Comparing predictors of conversion and decline in mild cognitive impairment.. Neurology, 75(3), pp. 230-38.

Lempel, A. and Ziv, J., 1976. On the complexity of finite sequence. IEEE Trans Inf Theory, Volume 22, p. 75-81.

Li, J., Yan, J., Liu, X. and Ouyang, G., 2014. Using Permutation Entropy to Measure the Changes in EEG Signals During Absence Seizures. Entropy, Volume 16, pp. 3049-3061.

Liang P, Wang Z, Yang Y, Jia X, and Li K., 2011. Functional disconnection and compensation in mild cognitive impairment: Evidence from DLPFC connectivity using resting-state fMRI. PLoS One, 6(7), e22153.

Liu, S., Guo, J., Meng, J., Wang, Z., Yao, Y., Yang, J., et al., 2016. Abnormal EEG Complexity and Functional Connectivity of Brain in Patients with Acute Thalamic Ischemic Stroke. Comput Math Methods M, 2016(2582478), pp. 1-9. 
Lobo, A., Ezquerra, J., Gomez Burgada, F., Sala, J. M., and Seva Diaz, A., 1979. Cognocitive mini-test (a simple practical test to detect intellectual changes in medical patients. Actas Luso Esp Neurol Psiquiatr Cienc Afines, 7(3), pp. 189-202.

López-Sanz, D., Bruña, R., Garcés, P., Camara, C. S.-R., Delgado, M. L., Montenegro, M., et al., 2016. Alpha band disruption in the AD-continuum starts in the Subjective Cognitive Decline stage: a MEG study. Scientific Reports, 6(37685), pp. 1-11.

López-Sanz, D., Bruña, R., Garces, P., Martin-Buro, M., Walter, S., Delgado, M., et al., 2017a. Functional Connectivity Disruption in Subjective Cognitive Decline and Mild Cognitive Impairment: A Common Pattern of Alterations.. Front. Aging Neurosci., 9(109), pp. 1-12.

López-Sanz, D., Garcés, P., Álvarez, B., Delgado-Losada, M. L., López-Higes, R., and Maestú, F., 2017b. Network Disruption in the Preclinical Stages of Alzheimer's Disease: From Subjective Cognitive Decline to Mild Cognitive Impairment. Int J Neural Syst, 27(8), p. 1750041.

Lutzenberger, W., Preissl, H. and Pulvermuller, F., 1995. Fractal dimension of electroencephalographic time series and underlying brain processes. Biol Cybern, Volume 73, pp. 477-482.

Maris, E. and Ostenveld, R., 2007. Nonparametric statistical testing of EEG- and MEG-data. J Neurosci Methods, Volume 164, pp. 177-190.

Meyer-Lindenber, A., 1996. The evolution of complexity in human brain development: An EEG study.. Electroencephalogr. Clin. Neuro., Volume 99, p. 405-411.

Nakamura A, Cuesta P, Fernández A, Arahata Y, Iwata K, Kuratsubo I, et al., 2018. Electromagnetic signatures of the preclinical and prodromal stages of Alzheimer's disease. Brain. 141(5):1470-85

Oostenveld, R., Fries, P., Maris, E. and Schoffelen, J.-M., 2011. FieldTrip: Open Source Software for Advanced Analysis of MEG, EEG, and Invasive Electrophysiological Data. Comput Intell Neurosci, 2011(156869), pp. 1-9.

Peter, J., Scheef, L., Abdulkadir, A., Boecker, H., Heneka, M. T., Wagner, M., et al., 2014. Gray matter atrophy pattern in elderly with subjective memory impairment. Alzheimers Dement, 10(1), pp. 99-108.

Petersen, R., 2004. Mild cognitive impairment as a diagnostic entity. J. Intern. Med., Volume 256, p. $183-194$.

Petersen, R. C., 2011. Clinical practice.Mild cognitive impairment. N Engl J Med Volume 364, pp. 2227-34.

Petersen, R. C. and Negash, S., 2008. Mild cognitive impairment: an overview. CNS spectrums, 13(1), pp. 45-53.

Pfeffer, R. I., Kurosaki, T. T., Harrah, C. H., Chance, J. M., and Filos, S., 1982. Measurement of functional activities in older adults in the community. J Gerontol, Volume 37, pp. 323-329.

Rabin, L., Smart, C. and Amariglio, R., 2017. Subjective Cognitive Decline in Preclinical Alzheimer's Disease. Annu. Rev. Clin. Psychol., Volume 13, pp. 369-396.

Reitan, R. M., 1958. Validity of the Trail Making Test as an indicator of organic brain damage. Percept Mot Skills, Volume 8, pp. 271-276.

Romei V, Thut G, Mok RM, Schyns PG and Driver J., 2012. Causal implication by rhythmic transcranial magnetic stimulation of alpha frequency in feature-based local vs. global attention. Eur J Neurosci 35(6):968-74.

Rosanova M, Casali A, Bellina V, Resta F, Mariotti M and Massimini M., 2009. Natural Frequencies of Human Corticothalamic Circuits. J Neurosci, 29(24):7679-85.

Rosen, W. G., Terry, R. D., Fuld, P. A., Katzman, R., and Peck, A., 1980. Pathological verification of ischemic score in differentiation of dementias. Ann. Neurol., Volume 7, pp. 486-488. 
Ryu, S. Y. Kwon, M.J., Lee, S.B., Yang, D.W., Kim, T.W., Song, I.U., et al., 2010. Measurement of precuneal and hippocampal volumes using magnetic resonance volumetry in Alzheimer's disease. J Clin Neurol., 6(4), pp. 196203.

Shumbayawonda, E., Fernández, A., Hughes, M. P. and Abasolo, D., 2017. Permutation Entropy for the Characterisation of Brain Activity Recorded with Magnetoencephalograms in Healthy Ageing. Entropy, 19(141), pp. 1-17.

Shumbayawonda, E., Tosun, P., Fernandez, A., Hughes, M., and Abasolo, D., 2018. Complexity Changes in Brain Activity in Healthy Ageing: A Permutation Lempel-Ziv Complexity Study of Magnetoencephalograms. Entropy, 20(506), pp. 1-16.

Sporns, O., Chialvo, D., Kaiser, M. and Hilgetag, C., 2004. Organization, development and function of complex brain network. Trends Cogn. Sci, 8(9), pp. 418-425.

Sporns, O., Tononi, G. and Kotte, R., 2005. The Human Connectome: A Structural Description of the Human Brain. PLoS Comput. Biol., 1(4), pp. 245-251.

Stam, C. J., van Straaten, E. C., Van Dellen, E., Tewarie, P., Gong, G., Hillebrand, A., et al., 2016. The relation between structural and functional connectivity patterns in complex brain networks. Int. J. Psychophysiol., Volume 103, pp. 149-160.

Stam, C., Nolte, G. and Daffertshofer, A., 2007. Phase lag index: assessment of functional connectivity from multi channel EEG and MEG with diminished bias from common sources.. Hum Brain Mapp, 28(11), pp. 1178-93.

Taulu, S. and Simola, J., 2006. Spatiotemporal signal space separation method for rejecting nearby interference in MEG measurements. Phys. Med. Biol., Volume 51, p. 1759-1768.

Tepest, R., Wang, L., Csernansky, J. G., Neubert, P., Heun, R., Scheef, L., and Jessen, F., 2008. Hippocampal surface analysis in subjective memory impairment, mild cognitive impairment and Alzheimer's dementia. Dement Geriatr Cogn Dis, 26(4), pp. 323-329.

Tononi, G., Edelman, G. M. and Sporns, O., 1998. Complexity and coherency: integrating information in the brain. Trends Cogn Sci, 2(12), pp. 474-484.

Utevsky, A. V., Smith, D. V. and Huetter, S. A., 2014. Precuneus Is a Functional Core of the Default-Mode Network. J. Neurosci., 34(3), p. 932-940..

Vos, S. J., van Rossum, I. A., Verhey, F., Knol, D. L., Soininen, H., Wahlund, L.-O., et al., 2013. Prediction of Alzheimer disease in subjects with amnestic and nonamnestic MCI. Neurology, 80(12), pp. 1124-32.

Wong, D. F., Rosenberg, P. B., Zhou, Y., Kumar, A., Raymont, V., Ravert, H. T., et al., 2010. In vivo imaging of amyloid deposition in Alzheimer disease using the radioligand 18F-AV-45 (florbetapir [corrected] F 18). J. Nucl. Med., 51(6), pp. 913-20.

Yesavage, J. A., Brink, T. L., Rose, T. L., Lum, O., Huang, V., Adey, M., and Leirer, V. O., 1982. Development and validation of a geriatric depression screening scale: A preliminary report. J Psychiatr Res., 17(1), pp. 37-49.

Figure captions

Fig 1: Illustration of the average global complexity values for each diagnosis group: controls (CN), subjective cognitive decline $(S C D)$ and mild cognitive impairment (MCI). In this figure, error bars show the standard error of the mean. 
Fig 2: Illustration of the location of the cluster with significantly different complexity values between the SCD and MCI groups ( $p=0.0404$ ) in the broadband analysis. The significant cluster was interpolated on a surface template (as seen in the lower part of the image), while, the coronal, sagittal and axial brain views are shown on interpolated T1 weighted images (as seen in the upper part of the image).

Fig 3: Illustration of the location of the clusters with significantly different complexity values between the CN and MCI groups ( $p=0.0362$ ) (left part) and in the SCD and MCI groups ( $p=0.0398)$ (right part) in the narrow band analyses. The significant cluster was interpolated on a surface template (as seen in the lower part of the image), while, the coronal, sagittal and axial brain views are shown on interpolated T1 weighted images (as seen in the upper part of the image).

Fig4: Illustration of the posterior cluster (shown in yellow) showing a significant correlation between complexity values in the low beta frequency range and right hippocampal volume $(p=0.0356)$ in the whole sample. 